DRAFT VERSION OCTOBER 19, 2016

Preprint typeset using LATEX style AASTeX6 v. 1.0

\title{
ON THE DIRECT CORRELATION BETWEEN GAMMA-RAYS AND PEV NEUTRINOS FROM BLAZARS
}

\author{
Shan GaO ${ }^{1}$, MARTin PohL ${ }^{1,2}$, WAlter Winter ${ }^{1}$ \\ ${ }^{1}$ Deutsches Elektronen-Synchrotron (DESY), Platanenallee 6, D-15738 Zeuthen, Germany \\ ${ }^{2}$ Institute of Physics and Astronomy, University of Potsdam, D-14476 Potsdam, Germany
}

\begin{abstract}
We study the frequently used assumption in multi-messenger astrophysics that the gamma-ray and neutrino fluxes are directly connected because they are assumed to be produced by the same photohadronic production chain. An interesting candidate source for this test is the flat-spectrum radio quasar PKS B1424-418, which recently called attention of a potential correlation between an IceCube PeV-neutrino event and its burst phase. We simulate both the multi-waveband photon and the neutrino emission from this source using a self-consistent radiation model. We demonstrate that a simple hadronic model cannot adequately describe the spectral energy distribution for this source, but a lepto-hadronic model with sub-dominant hadronic component can reproduce the multi-waveband photon spectrum observed during various activity phases of the blazar. As a conclusion, up to about 0.3 neutrino events may coincide with the burst, which implies that the leptonic contribution dominates in the relevant energy band. We also demonstrate that the time-wise correlation between the neutrino event and burst phase is weak.
\end{abstract}

\section{INTRODUCTION}

The diffuse flux of TeV-PeV neutrinos detected with IceCube indicates extraterrestrial neutrino emission from cosmic accelerators IceCube Collaboration (2013); Aartsen et al. (2013) of yet unknown nature. Among the prime source candidates are blazars, active galactic nuclei (AGN) featuring a relativistic jet roughly oriented along the line of sight to the observer. There is a rich literature modeling the neutrino emission from blazars and searching for positional and temporal correlations between the IceCube neutrino events and blazars (see e.g. Refs. Krauß et al. (2014); Padovani et al. (2016); Ahlers \& Halzen (2014); Dimitrakoudis et al. (2014); Petropoulou et al. (2016); Diltz \& Böttcher (2016); Righi et al. (2016); Halzen \& Kheirandish (2016) and references therein), as well as studying diffuse neutrino emission Murase et al. (2014); Dermer et al. (2014).

Blazars significantly contribute to the diffuse (extragalactic) $\gamma$-ray background Ajello et al. (2015). If these $\gamma$-rays originate from proton interactions, the energy budget will be in principle sufficient to account for the intensity of IceCube neutrinos Murase et al. (2013). However, recent stacking analyses using IceCube data suggest that AGN blazars at most contribute $7-27 \%$ of the observed neutrino intensity Glüsenkamp (2016); Kowalski (2016). Similar arguments apply to other promising source candidates, such as starburst galaxies Bechtol et al. (2015) and Gamma-Ray Bursts Abbasi et al. (2012); Aartsen et al. (2015). While

shan.gao@desy.de the origin of the astrophysical neutrinos is still unknown, the known constraints still permit that at least about ten percent of the observed neutrinos were produced by blazars Kowalski (2016), possibly even by a few particularly neutrino-bright blazars. Moreover, the information presented in different analyses seems partially contradictory, suggesting that substantial systematic uncertainties arise from generic assumptions concerning the spectral shape of the neutrino spectrum and the weighting schemes relating the neutrino luminosity to the $\gamma$-ray luminosity.

Blazars typically exhibit a two-hump structure in their spectral energy distribution (SED) Fossati et al. (1998); Ghisellini (2016). This structure has been successfully reproduced by both leptonic and hadronic models for a number of blazars Böttcher et al. (2013). In a leptonic model, the low-energy and high-energy humps of the SED are produced by the synchrotron emission from electrons and the inverseCompton scatter of soft photons (such as synchrotron photons from the same electrons), respectively. In a hadronic model, the primary electron-synchrotron generates the lowenergy hump as well, but secondaries from hadronic processes induced by $p \gamma$ interactions are responsible for the $\gamma$ ray emission. In the simplest cases, it is frequently used that in $p \gamma$ interactions the energy deposited in neutrinos (from $\pi^{ \pm}$ decays) is roughly comparable to that of $\gamma$-rays (from $\pi^{0} \mathrm{de}$ cays), which means that the neutrino and $\gamma$-ray fluxes are directly correlated. Note, however, that even in hadronic models, the leading mechanism to generate the second hump may be, for instance, synchrotron radiation of secondary electrons produced by the photohadronic interactions. In addi- 
tion, it may be possible that for certain astrophysical objects the emission in the second hump must be dominated by processes of purely leptonic origin. The study of this direct correlation is therefore the main motivation of this work.

One interesting test case for the direct neutrino- $\gamma$-ray correlation is the flat-spectrum radio quasar (FSRQ) PKS B1424-418. In Kadler et al. (2016), a positional and temporal coincidence between the $2-\mathrm{PeV}$ neutrino event (IceCube event 35, IC35, or "big bird") and a burst of PKS B1424-418 was reported. After analyzing the $\gamma$-ray fluences from blazars in the positional-uncertainty region of IC35 as well as the diffuse $\gamma$-ray emission, it was concluded that the burst of PKS B1424-418 had sufficient energy to account for the IceCube event, while the probability of a chance coincidence was around $5 \%$. The required neutrino production efficiency was obtained by scaling the measured neutrino output to the $\gamma$-ray fluence in the energy range from $5 \mathrm{keV}$ to $10 \mathrm{GeV}\left(10^{18.1}\right.$ $10^{24.4} \mathrm{~Hz}$ ) and found consistent with that theoretically expected on the grounds of flavor, spectral effects, and sourcepopulation selection. An important ingredient was the assumption that the SED in that respective energy range were of hadronic origin, implying our direct correlation. We study if this assumption can be maintained in a self-consistent ansatz. Note that while we focus on one astrophysical object in this study, our conclusions will be more profound, as this direct correlation is widely used in multi-messenger analyses, see e.g. Turley et al. (2016).

Hadronic model of blazars typically involve many parameters Böttcher et al. (2013), even in the simplest case: a parameter set for primary electrons, a set for protons, and a set for the bulk features of the emitting region, such as size (radius), magnetic-field strength, and Doppler factor, since none of these can be robustly derived from first principles or direct observations. The characteristics for the SEDs vary greatly among the blazars Ghisellini (2016), and therefore the bestfit parameters, even for purely leptonic models, exhibit large variations; an example are the leptonic models Finke et al. (2008) versus Tavecchio et al. (2013) for PKS B1424-418. Most neutrinos are produced in $p \gamma$ interactions near the $p \gamma$ threshold, and therefore the neutrino yield strongly depends on the density and spectrum of target photons. Even the simple assumption used in many studies, namely $L_{\nu} \propto L_{\gamma}$ (see e.g. Righi et al. (2016); Glüsenkamp (2016)), is questionable, and an accurate and self-consistent calculation of the SED and the neutrino spectrum is necessary. The calculation also needs to be efficient to permit scanning a large parameter space. In this paper we present such a code and use it together with analytical calculations to explore what kind of model and which model parameters provide a consistent description of both the SED and the rate of PeV neutrinos.

The paper is structured as follows: in Sec. 2 we introduce the general setup, and we use analytical calculations to determine the kind of model viable for PKS B1424-418; in Sec. 3 we introduce our numerical methods (see also App. A) and we present the results: the quality of the SED fit, the likelihood of having the observed neutrino event, and the corresponding parameters. We use cgs units throughout the paper, unless specified otherwise. All primed quantities are expressed in the blob-comoving frame.

\section{GENERAL ANALYSIS AND MODELS}

In this section we broadly list possible models and give generic constraints based on the characteristics of the SED for the source. The conclusions are derived using analytical and semi-analytical methods.

\subsection{Assumptions and List of Models}

We use a one-zone model consisting of an isotropic, homogeneous and spherical emission region, or blob, with radius $R_{\text {blob }}^{\prime}$, that moves relativistically with Doppler factor $\Gamma_{\text {bulk. }}$ Electrons and protons are injected with power-law spectra, $d^{2} n^{\prime} / d \gamma^{\prime} d t^{\prime}=K^{\prime} \gamma^{\prime \alpha}$ for $\gamma_{\text {min }}^{\prime}<\gamma^{\prime}<\gamma_{\text {max }}^{\prime}$, where $d^{2} n^{\prime} / d \gamma^{\prime} d t^{\prime}$ is the differential particle injection rate per volume, $\alpha$ the power-law index, $\gamma_{\min }^{\prime}$ and $\gamma_{\max }^{\prime}$ the minimum and maximum Lorentz factor of the particles, and $K^{\prime}$ is a normalization factor determined by the particle injection luminosity, $L_{\text {inj }}^{\prime}$. The injected electrons are henceforth referred to as primary electrons, whereas we denote as secondary electrons those created by hadronic interactions or $\gamma \gamma$ pair production. We allow primary electrons and protons to have separate parameter values for $L_{\text {inj }}^{\prime}, \gamma_{\min }^{\prime}, \gamma_{\max }^{\prime}$, and $\alpha$. The emission region is assumed to be filled with a homogeneous, randomly oriented magnetic field of strength $B^{\prime}$. Neutrinos and "optically-thin" photons can freely stream out of the blob on the timescale $t_{\mathrm{fs}}^{\prime}=4 R^{\prime} / 3 c$. For simplicity, we assume the escape rate for charged particles, in the slow cooling case, to be a fixed multiple of the free-streaming timescale, $t_{\mathrm{esc}}=t_{\mathrm{fs}} / f_{\mathrm{esc}}=10 t_{\mathrm{fs}}{ }^{1}$

The list of relevant interactions includes synchrotron emission and synchrotron self-absorption (SSA), inverse Compton (IC) scattering by both electrons and protons, $\gamma \gamma$ pair production and annihilation, Bethe-Heitler photo-pair production $p+\gamma \rightarrow p+e^{ \pm}(\mathrm{BH})$, and photo-hadronic $(p \gamma)$ interactions $\left(X+\gamma \rightarrow X^{\prime}+\pi\right)$, where $X$ and $X^{\prime}$ denotes either a proton or a neutron and $\pi$ includes charged or neutral pions. Secondary particles such as $\pi^{ \pm}$and $\mu^{ \pm}$can in principle radiate before they decay, but for the parameter values relevant to this study the effect is negligible.

\footnotetext{
${ }^{1}$ This is clearly an oversimplification as the modeling of the particle escape requires a detailed specification of the geometry, the boundary conditions, the magnetic-field configuration, etc., which is beyond the scope of the paper. The escape rate itself is likely to be energy-dependent to account for energy-dependent diffusion. In fact, synchrotron emission of escaped electrons may be responsible for the extended emission regions seen in VLBI radio data, and we include such a component with the corresponding escape rate in Fig. 4. Note that to some degree, the effect of energy-dependent escape can be compensated with an appropriate choice of the free parameters $L_{\mathrm{e}, \text { inj }}^{\prime}$ and $\alpha_{\mathrm{e}, \text { inj. }}$. The hadronic interactions are unaffected since the targetphoton distribution remains the same then.
} 
The low-frequency hump in the SED of a generic blazar extends from the radio band to the UV and in some cases even to the X-ray band; the high-frequency component can be observed from X-rays up to $\mathrm{TeV} \gamma$-rays. Here we discuss four scenarios that may in principle account for shape of the SED: the pure leptonic (SSC) model, the leptohadronic Synchrotron-Self-Compton (LH-SSC) model, the lepto-hadronic pion ( $\mathrm{LH} \pi$ ) model and the proton synchrotron (PSYN) model, which is a purely hadronic model. The defining features of these models are summarized in Tab. 1.

In both the SSC and LH-SSC model, the first hump is described by synchrotron emission of primary electrons, and the high-frequency component is due to inverse Compton scattering of those photons by the same electrons. The LHSSC model contains an additional hadronic component compared to the pure leptonic SSC model, which may fill the gap between the two humps (e.g., accounting for the X-ray emission from PKS B1424-418).

In the LH- $\pi$ model internally, the low-frequency hump is likewise described by synchrotron emission of primary electrons, whereas the second hump arises from hadronic processes. Depending on the parameters of the source, the dominant contribution to the second peak can be $\gamma$-rays from $\pi^{0}$ decays, synchrotron and inverse Compton radiation emitted by $e^{ \pm}$from $\pi^{ \pm}$decays, internal $\gamma \gamma$ annihilation, or protonpair production (Bethe-Heitler, $\mathrm{BH}$ ).

In the PSYN model, leptonic emission is sub-dominant at all wavebands. The first peak in the SED is attributed to proton-synchrotron radiation, and the second peak is produced by the same type of hadronic processes in the $\mathrm{LH} \pi$ model.

\subsection{Constraints on Models and Parameters from Semi-analytical Calculations}

While both the leptonic and the hadronic models have successfully explained the SED of a number of blazars, for example Mrk 421, 3C 279 etc., the unique combination of the SED with the PeV-neutrino information places stringent limits on the model of PKS B1424-418. Here we use analytical and semi-analytical calculations to demonstrate that neither the lepto-hadronic ( $\mathrm{LH} \pi$ ) nor the purely hadronic (PSYN) model can simultaneously explain the SED and the PeVneutrino event, leaving the SSC and LH-SSC models of the SED as the only viable contenders. Analytical arguments also give useful constraints on the parameter space.

PSYN model: In $\mathrm{p} \gamma$ interactions, the neutrino energy is roughly $5 \%$ of that of the parent proton, $E_{\nu} \sim 0.05 E_{p}$. For PKS B1424-418 at the redshift $z=1.522$, the Lorentz factor of the proton in the comoving frame can be written in terms of the bulk Lorentz factor of the blob $\Gamma$ and the neutrino energy in the observer frame $E_{\nu, \mathrm{PeV}}^{\mathrm{ob}} \equiv E_{\nu}^{\mathrm{ob}} / \mathrm{PeV}$ as

$$
\gamma_{\mathrm{p}}^{\prime} \sim 5 \times 10^{7} \Gamma^{-1} \mathrm{E}_{\nu, \mathrm{PeV}}^{\mathrm{ob}}
$$

If the low-energy peak is attributed to proton synchrotron emission, the peak frequency of the synchrotron emission, $\nu_{\mathrm{pk}, 1}$, obeys

$$
h \nu_{\mathrm{pk}, 1}^{\prime}=m_{\mathrm{e}} c^{2} \frac{m_{\mathrm{e}}}{m_{\mathrm{p}}}\left(\frac{B^{\prime}}{B_{\text {crit }}}\right) \gamma_{\mathrm{p}}^{\prime 2}
$$

where $B_{\text {crit }} \equiv 4.41 \times 10^{13} \mathrm{G}$ is the critical magnetic field. Combining Eqs. (1) and (2) provides a constraint on the magnetic field,

$$
\left.B^{\prime}\right|_{\mathrm{pk}, 1}=\left(7 \times 10^{-4} \mathrm{G}\right)\left(\frac{\nu_{\mathrm{pk}, 1}^{\mathrm{ob}}}{10^{14} \mathrm{~Hz}}\right)\left(\frac{\Gamma_{\mathrm{bulk}}}{10}\right)\left(\frac{E_{\nu}^{\mathrm{ob}}}{\mathrm{PeV}}\right)^{-2} .
$$

The peak frequency of the second hump in the SED relates to the peak energy of the secondary $e^{ \pm}$that result from either $\pi^{ \pm} \rightarrow e^{ \pm}$decay or from $\pi^{0} \rightarrow \gamma \gamma \rightarrow e^{ \pm}$reactions. It will be shown later that for PKS B1424-418, up to one generation of $e^{ \pm}$cascade is expected, so that for both channels the energy of $e^{ \pm}$is $E_{\mathrm{e}} \sim 0.05 E_{\mathrm{p}}$. Reproducing the second hump with the observed peak frequency $\nu_{\mathrm{pk}, 2}^{\mathrm{ob}}$ from synchrotron emission of the secondary pairs requires a magnetic-field strength

$$
\left.B^{\prime}\right|_{\mathrm{pk}, 2}=\left(3 \times 10^{-2} \mathrm{G}\right)\left(\frac{\nu_{\mathrm{pk}, 2}^{\mathrm{ob}}}{10^{23} \mathrm{~Hz}}\right)\left(\frac{\Gamma_{\text {bulk }}}{10}\right)\left(\frac{E_{\nu}^{\mathrm{ob}}}{\mathrm{PeV}}\right)^{-2} \text {. }
$$

The value $\left.B\right|_{\mathrm{pk}, 2}$ is clearly incompatible with $\left.B\right|_{\mathrm{pk}, 1}$, which means that the PSYN model is not viable.

LH- $\boldsymbol{\pi}$ model: This scenario is slightly more complicated, but a few generic conditions must be met:

1. The proton synchrotron flux must not exceed that of synchrotron radiation of primary electrons.

2. The $Y_{\mathrm{SSC}}$ parameter, defined as the power ratio of synchrotron-self Compton emission to synchrotron emission, must not exceed unity for this source (otherwise the model becomes the SSC model).

3. The observed peak frequency of the high-energy hump in the SED $\nu_{\mathrm{pk}, 2}^{\mathrm{ob}} \sim 10^{23} \mathrm{~Hz}$ must be consistent with the characteristic energy of the secondary $e^{ \pm}$from $\mathrm{p} \gamma$ interactions $E_{\mathrm{e}} \sim 0.05 E_{\mathrm{p}}$, with $E_{\mathrm{p}}$ determined from the neutrino energy $E_{\nu, \mathrm{PeV}}^{\mathrm{ob}}$.

4. The emission from pairs by the Bethe-Heitler process must not overshoot the observation.

For this calculation, the SED is approximated by four segments of power-law spectra. All four constraints are displayed in Fig. 1 for a blob radius $R_{\text {blob }}^{\prime}=10^{18} \mathrm{~cm}$. When $R_{\text {blob }}^{\prime}$ increases, the boundaries of the grey and green regions (corresponding to constraints 1 and 2, respectively) move towards the lower-left corner of the panel, whereas the regions defined by constraints 3) and 4) are independent of the $R_{\text {blob }}^{\prime}$. There is no region of overlap for all four constraints, whatever the value of $R_{\text {blob }}^{\prime}$, which rules out the LH- $\pi$ model for PKS B1424-418. 


\begin{tabular}{|c|c|c|c|}
\hline & $\begin{array}{l}\text { First peak } \\
(\mathrm{eV}-\mathrm{keV})\end{array}$ & $\begin{array}{l}\text { Middle range } \\
(\mathrm{keV}-\mathrm{MeV})\end{array}$ & $\begin{array}{l}\text { Second peak } \\
(\mathrm{MeV}-\mathrm{TeV})\end{array}$ \\
\hline $\begin{array}{c}\text { SSC } \\
\text { (Pure leptonic) }\end{array}$ & $\begin{array}{c}\mathbf{L} \\
\text { Primary } e^{-} \text {synchrotron }\end{array}$ & $\begin{array}{c}\mathbf{L} \\
\text { SSC }\end{array}$ & $\begin{array}{c}\mathbf{L} \\
\text { SSC }\end{array}$ \\
\hline $\begin{array}{c}\text { LH-SSC } \\
\text { (Lepto-hadronic) }\end{array}$ & $\begin{array}{c}\mathbf{L} \\
\text { Primary } e^{-} \text {synchrotron } \\
\end{array}$ & $\begin{array}{c}\mathbf{H} \\
\text { Secondary leptonic }\end{array}$ & $\begin{array}{c}\mathbf{L} \\
\text { SSC by primary } e^{-}\end{array}$ \\
\hline $\begin{array}{c}\text { LH- } \boldsymbol{\pi} \\
\text { (Lepto-hadronic) }\end{array}$ & $\begin{array}{c}\mathbf{L} \\
\text { Primary } e^{-} \text {synchrotron }\end{array}$ & $\begin{array}{c}\mathbf{H} \\
\text { Secondary leptonic }\end{array}$ & \begin{tabular}{l}
\multicolumn{1}{c}{$\mathbf{H}$} \\
Secondary leptonic or $\gamma$-rays \\
from direct $\pi^{0}$ decay
\end{tabular} \\
\hline $\begin{array}{c}\text { PSYN } \\
\text { (Pure hadronic) }\end{array}$ & $\begin{array}{c}\mathbf{H} \\
\text { Proton synchrotron }\end{array}$ & $\begin{array}{c}\mathbf{H} \\
\text { Secondary leptonic }\end{array}$ & $\begin{array}{c}\mathbf{H} \\
\text { Same as above }\end{array}$ \\
\hline
\end{tabular}

Table 1. List of models. In the table, "L"="Leptonic" and "H"="Hadronic". "LH" is the abbreviation for "lepto-hadronic", which is a mixture of leptonic and hadronic components.

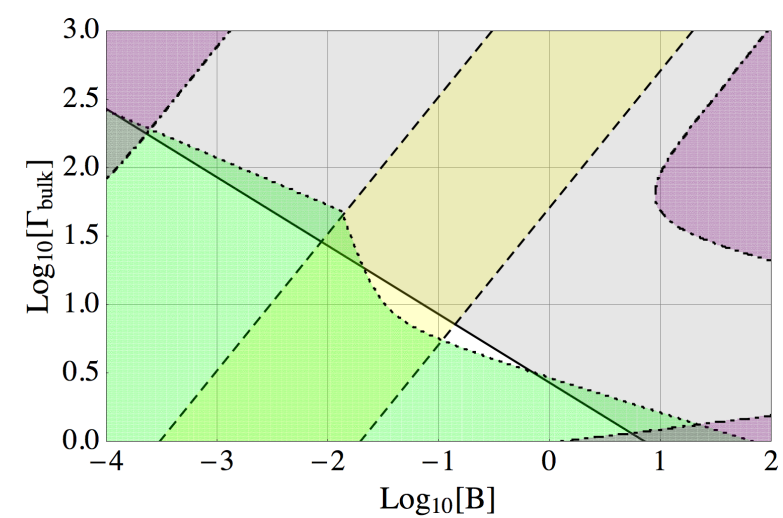

Figure 1. Allowed parameter regions for the LH $\pi$ model ( $c f$., Sec. 2.2). Green: lower-left region with dotted boundary, correponding to constraint 1; Grey: upper-right region limited by the solid line, constraint 2; Yellow area between the dashed parallel lines, constraint 3; Purple: the three separate regions formed by dot-dashed boundaries.

SSC and LH-SSC models: In the Thomson scattering regime, the frequency of the scattered photon is $\nu_{\mathrm{pk}, 2}^{\prime} \simeq$ $\gamma_{\mathrm{e}}^{\prime 2} \nu_{\mathrm{pk}, 1}^{\prime}$. Scattering proceeds in the Klein-Nishina regime for electron Lorentz factors $\gamma^{\prime} \gtrsim \gamma_{\mathrm{KN}}^{\prime}$, where $\gamma_{\mathrm{KN}}^{\prime} h \nu_{\mathrm{pk}, 1}^{\prime} \sim m_{\mathrm{e}} c^{2}$. Combining the two expressions, we find the following relationship among the Lorentz factor of the accelerated electrons in the comoving frame $\gamma_{\mathrm{e}}^{\prime}$ and the SED parameters of PKS B1424-418:

$$
\gamma_{\mathrm{e}}^{\prime}=0.007 \gamma_{\mathrm{KN}}^{\prime}\left(\frac{\nu_{\mathrm{pk}, 1}^{\mathrm{ob}}}{10^{14} \mathrm{~Hz}}\right)\left(\frac{\nu_{\mathrm{pk}, 2}^{\mathrm{ob}}}{10^{23} \mathrm{~Hz}}\right)\left(\frac{\Gamma}{10}\right)^{-1}<\gamma_{\mathrm{KN}}^{\prime} .
$$

We conclude that the inverse Compton scattering of primary electrons is always Thomson scattering.

The comoving photon density can be written as

$$
u_{\mathrm{ph}}^{\prime}=\left(\frac{d}{R_{\mathrm{blob}}}\right)^{2}\left(\frac{1+z}{\Gamma^{2}}\right)^{2} \frac{\nu F_{\nu}}{c} .
$$

Here $d=4.477 \mathrm{Gpc}$ is the comoving radial distance of the source (For $z=1.522$ and a flat $\Lambda \mathrm{CDM}$ universe with $\Lambda=$ $0.7)$, and $u_{\mathrm{B}}^{\prime}=B^{\prime 2} / 8 \pi$ is the comoving magnetic-field energy density. We can express the constraints on $B^{\prime}, R_{\mathrm{blob}}^{\prime}$, and $\Gamma$ in terms of observed quantities as

$$
\begin{aligned}
R_{\mathrm{blob}}^{\prime} \Gamma & \approx\left(1.4 \times 10^{19} \mathrm{~cm}\right) Y_{\mathrm{SSC}}^{-1 / 2} \\
& \left(\frac{\left.\nu F_{\nu}\right|_{\mathrm{pk}, 1} ^{\mathrm{ob}}}{10^{-11} \mathrm{erg} \mathrm{cm}^{-2} \mathrm{~s}^{-1}}\right)^{1 / 2}\left(\frac{\nu_{\mathrm{pk}, 1}^{\mathrm{ob}}}{10^{14} \mathrm{~Hz}}\right)^{-2}\left(\frac{\nu_{\mathrm{pk}, 2}^{\mathrm{ob}}}{10^{23} \mathrm{~Hz}}\right),
\end{aligned}
$$

and

$$
B^{\prime} \approx\left(9.2 \times 10^{-3} \mathrm{G}\right)\left(\frac{\nu_{\mathrm{pk}, 1}^{\mathrm{ob}}}{10^{14} \mathrm{~Hz}}\right)^{2}\left(\frac{\nu_{\mathrm{p}, 2}^{\mathrm{ob}}}{10^{23} \mathrm{~Hz}}\right)^{-1}\left(\frac{\Gamma}{10}\right)^{-1}
$$

where

$$
Y_{\mathrm{SSC}} \approx \frac{\nu^{\prime} u_{\nu}^{\prime}\left(\nu_{\mathrm{pk}, 1}^{\prime}\right)}{u_{\mathrm{B}}^{\prime}} \approx \frac{\left.\nu \mathrm{F}_{\nu}\right|_{\mathrm{pk}, 2}}{\left.\nu \mathrm{F}_{\nu}\right|_{\mathrm{pk}, 1}} \approx 10
$$

and $u_{\nu}^{\prime}$ is the differential energy density of photons in the comoving frame. The scaling values of the observed quantities are typical for the SED of PKS B1424-418 (cf., Fig.4). Therefore Eq. (7) implies that the blob radius be large, on the order of a light-year. The magnetic-field strength must be rather low, suggesting that the blob is located far away from the central engine, beyond the broad-line region and the dusty torus (see also Tavecchio et al. (2013)). This scenario justifies that we neglect inverse Compton scattering of external photons.

The optical depth of MeV-band $\gamma$-rays to pair production, $\tau_{\gamma \gamma}$, can be estimated as

$$
\begin{aligned}
\tau_{\gamma \gamma} & \approx \frac{\mathrm{L}^{\prime}}{R_{\mathrm{blob}}^{\prime}} \frac{\sigma_{T}}{4 \pi m_{e} c^{3}} \\
& \approx 3 \times 10^{-5}\left(\frac{\nu F_{\nu}^{o b}}{10^{-11} \mathrm{erg} \mathrm{cm}^{-2} \mathrm{~s}^{-1}}\right)\left(\frac{\Gamma}{10}\right)^{-4}\left(\frac{R_{\text {blob }}^{\prime}}{10^{18} \mathrm{~cm}}\right)^{-1},
\end{aligned}
$$

indicating that $\mathrm{MeV} \gamma$-rays can escape the source. A numerical calculation on the optical depth shows that $\tau_{\gamma \gamma}$ approaches unity for multi- $\mathrm{TeV}$ to $\mathrm{PeV} \gamma$-rays for typical model parameters for PKS B1424-418, which is consistent with one cascade generation of these $\gamma$-rays. 


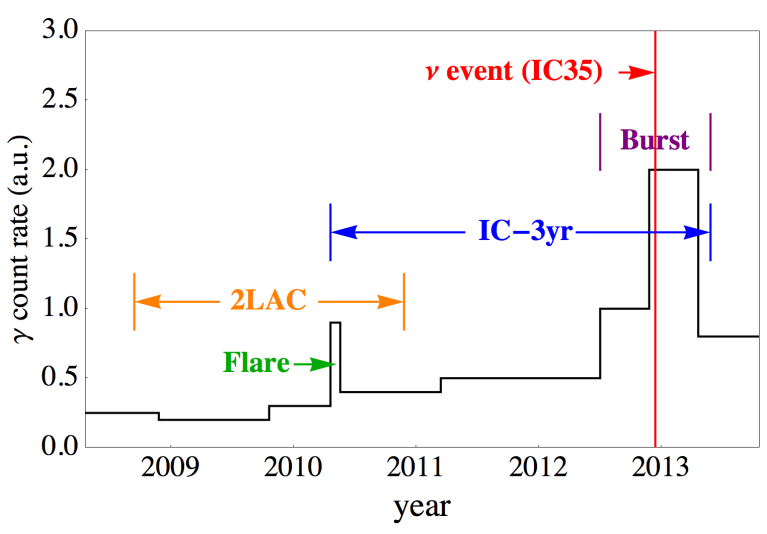

Figure 2. An illustration of the $\gamma$-ray count rate as a function of time together with a definition of the 2LAC, IC-3yr, and Burst phases of PKS B1424-418. Note that the count rate is meant for illustrative purposes only and does not accurately reflect the data (see Figure 1 of Kadler et al. (2016) for the bi-weekly binned $\gamma$-ray light curve).

The position and flux of the high-energy peak in the SED need to be explained by the SSC model. Determining the permitted abundance of hadrons as well as the detailed fitting of the entire SED and the neutrino data require the numerical modeling of the source, which is discussed in the following section.

PKS B1424-418 exhibits significant time variabilities over a wide range of scales, as other FSRQs and BL Lacs do. Variability timescales $t_{\mathrm{var}}^{\mathrm{ob}}$ as short as a month are consistent with the causality argument $t_{\mathrm{var}}^{\mathrm{ob}} \sim R_{\mathrm{blob}}^{\prime}(1+z) / \Gamma c$ and the constraint set by Eq. (7) for a blob size $R_{\text {blob }}=7.5 \times 10^{17} \mathrm{~cm}$ and a bulk Lorentz factor $\Gamma=35$, for which $t_{\mathrm{var}}^{\mathrm{ob}} \simeq 3$ weeks. ${ }^{2}$ Faster variability has been observed on timescales shorter than a day, which may be explained by compact substructures in the jet, such as re-collimation Bromberg \& Levinson (2009), "jet in a jet" scenarios, or magnetic reconnection Giannios et al. (2009); Giannios (2013). In any case, explaining this very fast variability is beyond the scope of this paper.

\section{NUMERICAL SED MODEL AND CONSEQUENCES FOR THE NEUTRINO PRODUCTION}

We present our numerical simulation results for the SSC and LH-SSC scenarios here, which were found to be preferable in the previous section. After discussing our methods, we will first discuss the Burst phase, and then show a selfconsistent picture for the evolution of the blazar over the full studied timeline.

\subsection{Methods}

We simulate time-dependent particle spectra for $e^{ \pm}, p, n$, $\gamma$, and $\nu_{\alpha}$ ( $\alpha$ denotes the neutrino flavor) by numerically solving the time-dependent differential equation system describing these species, see App. A for details. Our list of

\footnotetext{
${ }^{2}$ This choice of $\Gamma$ is also in line with the best-fit spectra by numerical simulations over the parameter space.
}

input parameters and assumptions is summarized in Tab. 2, which we describe in greater detail in this and the next sections.

The dynamical SEDs of PKS B1424-418 reported in Kadler et al. (2016) are categorized into four phases: 1) flare in 2010, lasting about 1 month; 2) 2LAC phase, from 2008.8 to 2010.9; 3) IC-3yr period, from 2010.5 to 2013.5, the first three years of IceCube observation; 4) Burst phase, from 2012.5 to 2013.3, when the source experienced a longlasting high-flux phase in $\gamma$-rays. Fig. 2 provides a visual timeline including the time-averaged $\mathrm{GeV}$-band $\gamma$-ray flux. A 2-PeV neutrino event in IceCube (IC35, also dubbed "big bird") was observed on Dec. 4, 2012, during phases 3) and 4), with a position consistent with that of PKS B1424-418. The SEDs shown in Kadler et al. (2016) are based on timeaveraged spectra from each phase. In this paper, the flare phase is ignored since its duration was too short to result in a neutrino fluence comparable with that of other phases. ${ }^{3} \mathrm{We}$ therefore focus on three phases (2LAC, IC-3yr, and Burst), as indicated in Fig. 2, which can be, in spite of some overlap, interpreted as the time-dependent evolution of the AGN. We will first simulate the phases independently, and then interpret the evolution (changes) of the parameters.

For each phase, the SED and neutrino spectra are modeled by a steady-state solution to Eq. (A1). Under the SSC and LH-SSC scenarios, the first peak of the SED is described as synchrotron emission from primary $e^{-}$, and the $\gamma$-rays are described as SSC emission from the same $e^{-}$population (see Tab. 1). After fixing a few global parameters such as $R_{\text {blob }}$ and $\Gamma_{\text {bulk }}$, the following two-step simulations are performed for each of the three phases of PKS B1424-418 (2LAC, IC3yr, and Burst): 1) Use leptonic simulations $\left(\eta_{b} \equiv 0\right)$ to find the best-fit parameters of primary $e^{-}$for the low-energy hump and $\gamma$-ray band $\left(10^{22}-10^{25} \mathrm{~Hz}\right)$. 2) Inject protons, until their spectrum reaches a steady state to find the total SED and neutrino spectrum.

In step 1, with leptonic simulations, the following parameter space is scanned: $L_{\mathrm{e}, \mathrm{inj}} \otimes \gamma_{\mathrm{e}, \min }^{\prime} \otimes \gamma_{\mathrm{e}, \max }^{\prime} \otimes \alpha_{\mathrm{e}, \mathrm{inj}} \otimes B^{\prime}$. The best-fit parameters are obtained by $\chi^{2}$-minimization. ${ }^{4}$

In step 2, including protons, the SED and the neutrino spectrum are calculated for a logarithmic $\eta_{\mathrm{b}} \otimes E_{\mathrm{p}, \text { max }}^{\prime}$ parameter grid for each phase of PKS B1424-418. Here $\eta_{\mathrm{b}}$ is the proton-to-electron luminosity ratio (baryonic loading) at injection, $\eta_{\mathrm{b}}=L_{\mathrm{p}, \text { inj }} / L_{\mathrm{e}, \text { inj }}$, and $E_{\mathrm{p} \text {, max }}^{\prime}$ is the maximum proton energy. Each point of the grid corresponds to a unique combination of $\eta_{\mathrm{b}}$ and $E_{\mathrm{p}, \max }$ and therefore an independent simulation. The entire grid has a resolution of $400 \times 80$ for each

\footnotetext{
${ }^{3}$ Even for the most optimistic estimates in Kadler et al. (2016), the expected neutrino count is far below 1.0, consistent with the null detection of neutrinos during this phase.

${ }^{4}$ For simplicity, we have used a constant relative uncertainty for each data point. This procedure corresponds to fitting the data points by bare eye, and cannot be interpreted statistically.
} 


\begin{tabular}{|c|c|c|c|}
\hline & Group & Symbol & Definition \\
\hline \multirow{3}{*}{ Parameters } & Global & $\begin{array}{c}R_{\text {blob }}^{\prime} \\
f_{\text {esc }} \\
\Gamma_{\text {bulk }}\end{array}$ & $\begin{array}{l}\text { Comoving radius of blob, fixed to } 7.5 \times 10^{17} \mathrm{~cm} \\
e^{ \pm} \text {and } p \text { escape fraction, fixed to } 1 / 10 \\
\text { bulk Lorentz factor of the blob fixed to } 35\end{array}$ \\
\hline & Leptonic & $\begin{array}{c}B^{\prime} \\
L_{\mathrm{e}, \text { inj }} \\
\gamma_{\mathrm{e}, \min }^{\prime} \\
\gamma_{\mathrm{e}, \max }^{\prime} \\
\alpha_{\mathrm{e}, \mathrm{idx}}^{\prime}\end{array}$ & $\begin{array}{l}\text { Magnetic field strength, blob frame } \\
\text { Injection luminosity of primary } e^{-}, \mathrm{AGN} \text { frame } \\
\text { Minimum Lorentz factor of primary } e^{-}, \text {blob frame } \\
\text { Maximum Lorentz factor of primary } e^{-}, \text {blob frame } \\
\text { Power law index of injected primary } e^{-}\end{array}$ \\
\hline & Hadronic & $\begin{array}{c}\alpha_{\mathrm{p}, \mathrm{idx}}^{\prime} \\
\eta_{\mathrm{b}} \\
E_{p, \max }^{o b} \\
\end{array}$ & $\begin{array}{l}\text { Power law index of injected protons, fixed to }-2.0 \\
\text { Luminosity ratio } p \text { to } e^{-} \text {at injection, i.e., } \eta_{\mathrm{b}} \equiv L_{\mathrm{p}, \text { inj }} / L_{\mathrm{e}, \text { inj }} \\
\text { Maximal energy of injected protons, observer frame }\end{array}$ \\
\hline Notations & $P_{0,1,0}\left(E_{\mathrm{p}}\right.$ & $\left.\operatorname{nax}, \eta_{\mathrm{b}}\right)$ & $\begin{array}{l}\text { Probability to observe } 0,1,0 \text { neutrino events in the } 0.5-1.6 \mathrm{PeV}, 1.6-2.4 \mathrm{PeV} \text { and } \\
>2.4 \mathrm{PeV} \text { bands in IceCube, respectively, as a function of } E_{\mathrm{p}, \max } \text { and } \eta_{\mathrm{b}} \\
\text { Maximum value of } P_{0,1,0}\left(E_{\mathrm{p}, \max }, \eta_{\mathrm{b}}\right) \text { in the parameter space, named as "neutrino } \\
\text { best-fit" } \\
\text { Total neutrino luminosity, including all flavors } \\
\gamma \text {-ray luminosity integrated over the frequency band } 10^{18.1} \sim 10^{24.4} \mathrm{~Hz} \\
\text { SED best-fit mark } \\
\text { Neutrino best-fit mark, global } \\
\text { Neutrino best-fit under the constraint of SED being reproduced within } 3 \sigma \\
\text { confidence } \\
\text { Joint best-fit mark, for SED and neutrino } \\
\text { Neutrino flux to expect one }>0.5 \text { PeV event during } 2 \mathrm{LAC} \text { phase, assuming the } \\
\text { same effective area of IceCube as in IC-3yr and Burst phase (see Fig. 5) }\end{array}$ \\
\hline
\end{tabular}

Table 2. List of parameters and notations.

phase, requiring a total of 96000 hadronic simulations.

The global parameters $R_{\text {blob }}^{\prime}=7.5 \times 10^{17} \mathrm{~cm}$ and $\Gamma_{\text {bulk }}=$ 35 are chosen according to Eq. (7), including a variability time $t_{\mathrm{var}}<1$ month. The energy-independent escape rate for $e^{-}$and $p$ is assumed to be $t_{\mathrm{e}, \mathrm{p}}^{-1}=0.1 t_{\mathrm{fs}}^{-1}$, which is an order magnitude slower than the free-streaming escape rate (that of the neutrinos). We also use a fixed power law injection index $\alpha_{\mathrm{p} \text {,inj }}=-2.0$ for proton injection, where the results are not very sensitive to this value.

TANAMI VLBI data of PKS B1424-418 indicate an angular size of a few milli-arcsecond for the emission region in the radio band, which translates into a physical size of about $10^{19} \mathrm{~cm}$ - which is larger than that of the blob. The extended VLBI component can be interpreted as synchrotron emission from electrons that escaped from the blob into an extended region of size $R_{\text {ext }} \sim 2.0 \times 10^{19} \mathrm{~cm}$ that is filled with weaker magnetic field $B_{\text {ext }}^{\prime} \simeq 0.05 B_{\text {blob }}^{\prime}$. We assume that the radio data are fitted by synchrotron emission from electrons that escaped the blob into an extended region using these parameters. All other contributions to the SED come from the blob.

\subsection{SED Model and Neutrino Production in the Burst Phase}

In this subsection, we focus on the Burst phase and the relationship to the potentially observed neutrino event during

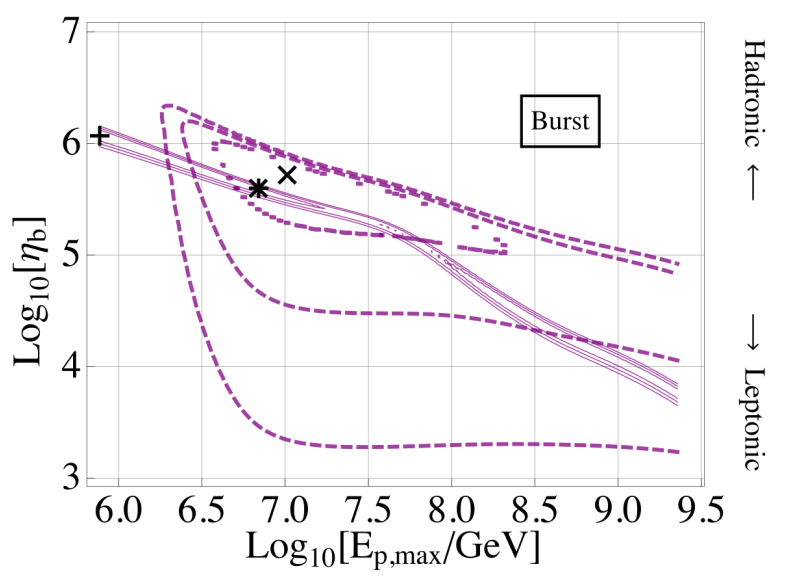

Figure 3. Fit to SED (solid) and neutrino observations (dashed) in terms of maximal proton energy and baryonic loading for the Burst phase. The solid and dashed contours correspond to the reduced $\chi^{2}$ of the SED fit and the probability $P$ to observe the IceCube neutrino event IC35 during the IC-3yr and Burst phase of PKS B1424-418, respectively (solid curves are the boundaries of $1 \sigma, 2 \sigma$, and $3 \sigma$ regions for the SED fit, while dashed curves are iso-probability contours for $P / P_{\nu, \max }=0.32,0.05,0.003$ - see main text for details). The symbols,$+ \times$ and $*$ mark the best-fit parameters of the SED fit, the neutrino fit, and the joint fit, respectively.

that phase.

For each parameter-space simulation, we perform indepen- 
dent optimizations. First, we adapt our model to the SED and find the best fit and confidence regions by calculating the reduced $\chi^{2}$-values. This SED best-fit is marked with the symbol " +". Then with the predicted neutrino spectrum from each simulation, we calculate the probability, $P_{010}\left(E_{\mathrm{p}, \max }, \eta_{\mathrm{b}}\right)$ to observe from PKS B1424-418 with IceCube 0,1, and 0 neutrino events in the $(0.5-1.6),(1.6-2.4)$, and $>2.4 \mathrm{PeV}$ energy bands ${ }^{5}$, respectively, as was indeed observed. The best adaption to the neutrino data without regard of the SED is referred to by the symbol " $\times$ ", and its fit probability is denoted as $P_{\nu, \max }$. The joint best-fit point, by maximizing the joint probability of the SED and neutrino fit, is marked with the symbol "*⿻丷木"⿱

We find that both SED and neutrino fits independently prefer large baryonic loadings for the Burst phase, which may point towards a baryonically loaded burst. At the neutrino and joint best-fit points in Fig. $3(\times, *)$, the associated proton maximum energies are around $10 \mathrm{PeV}$, which is consistent with the observed PeV neutrino event. However, the maximal proton flux at the neutrino best-fit $\times$ is in tension with the X-ray data, which we will demonstrate below.

We show the SED for the SED best-fit (+), the joint bestfit (*) and the neutrino best-fit $(\times)$ in the different rows of Fig. 4, where the individual contributions from purely leptonic and hadronic processes are depicted as well. From the lower panel, we clearly observe that the SED is in tension with data in the X-ray energy range. On the other hand, the SED is described reasonably well for the other two fit points. Note that the figure shows the radio data from the extended emission region as dashed curve $2 .{ }^{6}$

As a next step, we address the question whether the observed neutrino event can come from the Burst phase from PKS B1424-418, as reported in Kadler et al. (2016). One test of this hypothesis is energetics, i.e., in Kadler et al. (2016), the neutrino luminosity was directly related to the section of the SED we marked " $L_{\gamma}$ " in Fig. 4 . One can easily see that this energy range is dominated by leptonic processes (1) in our model, contrary to what has been assumed in Ref. Kadler et al. (2016) (for which the hadronic processes 2-4 would need to dominate that energy range).

We list the fractional contributions of the relevant channels during the Burst phase in that energy range in Tab. 3 for the different fit assumptions, where one can clearly read off that the SSC contribution dominates independent of the fit. We also show the neutrino-to- $\gamma$-ray ratio $L_{\nu} / L_{\gamma}$, which is order $10 \%$ for the models fitting the SED, and order $30 \%$

\footnotetext{
5 In each band, the expected number of neutrino events follows a Poisson distribution.

6 The SSC component from the extended region is about one order of magnitude weaker than the corresponding part of SED from the blob, due to a much lower number density of target photons in the extended region. This component is omitted in Fig. 4 in order to make the hadronic components (2)-(4) more identifiable in the figure.
}

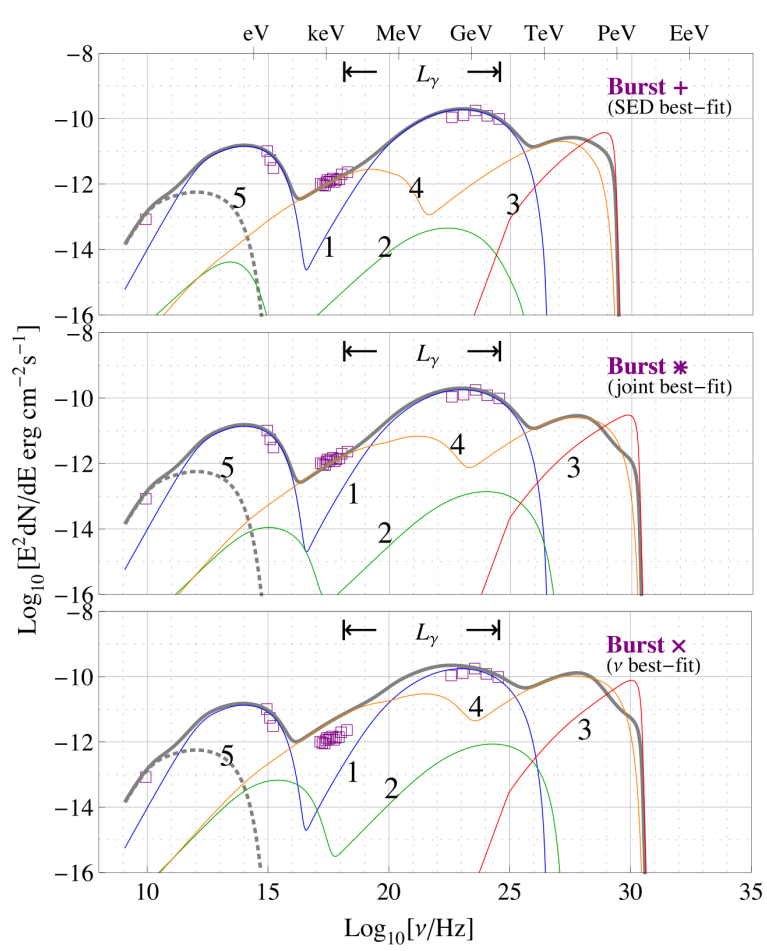

Figure 4. SEDs for the Burst phase corresponding to the SED (upper panel), joint neutrino-SED (middle panel), and neutrino (lower panel) best-fits (see Fig. 3). The curves are labeled as follows: grey thick line - total SED; blue (1) - emission from primary electrons; green (2) - emission from pairs generated via Bethe-Heitler process; red (3) - $\gamma$ ray injection spectrum from $\pi^{0}$ decay; orange (4) - emission from secondary pairs, generated via channels $p \gamma \rightarrow \pi^{0} \rightarrow \gamma \gamma \rightarrow e^{ \pm}$and $p \gamma \rightarrow \pi^{ \pm} \rightarrow \mu^{ \pm} \rightarrow e^{ \pm}$; gray dashed (5) - synchrotron emission from the extended region by electrons escaping the blob. For curves (1),(2) and (4), the left hump comes from the synchrotron emission, while the right hump comes from the inverse Compton component. Note that curves (2)-(4) are of hadronic origins. The $\gamma \gamma$-absorption effect in the source becomes significant above $\sim 100 \mathrm{TeV}$, which is manifested in the suppression of the total SED in that energy band. The data points here and in Fig. 6 are provided by Kadler et al. (2016) (processed from data by Fermi-LAT, Swift-XRT/UVOT, SMARTS, and the LBA, etc; see the supplementary material for the data analysis methods in Kadler et al. (2016)).

for the neutrino fit. These numbers are to be interpreted as an additional "theory" correction factor in addition to those included in Kadler et al. (2016) (independent of the spectral correction in Kadler et al. (2016), which would reduce this number). They reflect the fact that hadronic processes only dominate in a small portion of the energy range marked " $L_{\gamma}$ " in Fig. 4, considering that the second hump cannot be dominated by hadronic processes. Note, however, that $L_{\nu} / L_{\gamma}$ is higher than what one would expect from the non-SSC ontributions in Tab. 3, as hadronic components contribute significantly to the SED outside the considered energy range. In Kadler et al. (2016), the predicted number of PeV neutrinos is 1.6, assuming that the entire second hump is generated from hadronic processes. From our model, this needs to be 


\begin{tabular}{|l|l|l|rrrr|r|}
\hline Phase & Case & Figure & $\mathrm{SSC} / L_{\gamma}$ & $\mathrm{BH} / L_{\gamma}$ & $\pi^{0} / L_{\gamma}$ & $\pi^{ \pm} / L_{\gamma}$ & $L_{\nu} / L_{\gamma}$ \\
\hline \multirow{4}{*}{ Burst } & + (SED best-fit) & Fig. 4 (top) & 0.98 & 0.00025 & 0.011 & 0.0066 & 0.13 \\
& * (joint best-fit) & Fig. 4 (middle) & 0.95 & 0.00052 & 0.032 & 0.012 & 0.13 \\
& $\times(\nu$ best-fit) & Fig. 4 (bottom) & 0.82 & 0.0025 & 0.12 & 0.056 & 0.33 \\
\hline
\end{tabular}

Table 3. Luminosity contributions to the band $10^{18.1} \sim 10^{24.4} \mathrm{~Hz}(5 \mathrm{keV}$ to $10 \mathrm{GeV})$ from various interaction channels and the total neutrino luminosity, separately listed for the three best-fit cases of the Burst phase. All numbers are normalized to the total $\gamma$-ray luminosity, $L_{\gamma}$, defined as the integrated luminosity over the band $10^{18.1} \sim 10^{24.4} \mathrm{~Hz}$ and $L_{\nu}$ is the all-flavor, total neutrino luminosity (over the entire energy axis). "SSC" corresponds to the inverse Compton emission by primary $e^{-}$in this band. "BH" corresponds to the emission by pairs generated via the Bethe-Heitler process. The symbol " $\pi^{0}$ " refers to the emission by pairs created via $p \gamma \rightarrow \pi^{0} \rightarrow \gamma \gamma \rightarrow e^{ \pm}$while " $\pi^{ \pm}$" denotes the emission by pairs via the $p \gamma \rightarrow \pi^{ \pm} \rightarrow \mu^{ \pm} \rightarrow e^{ \pm}$channel.
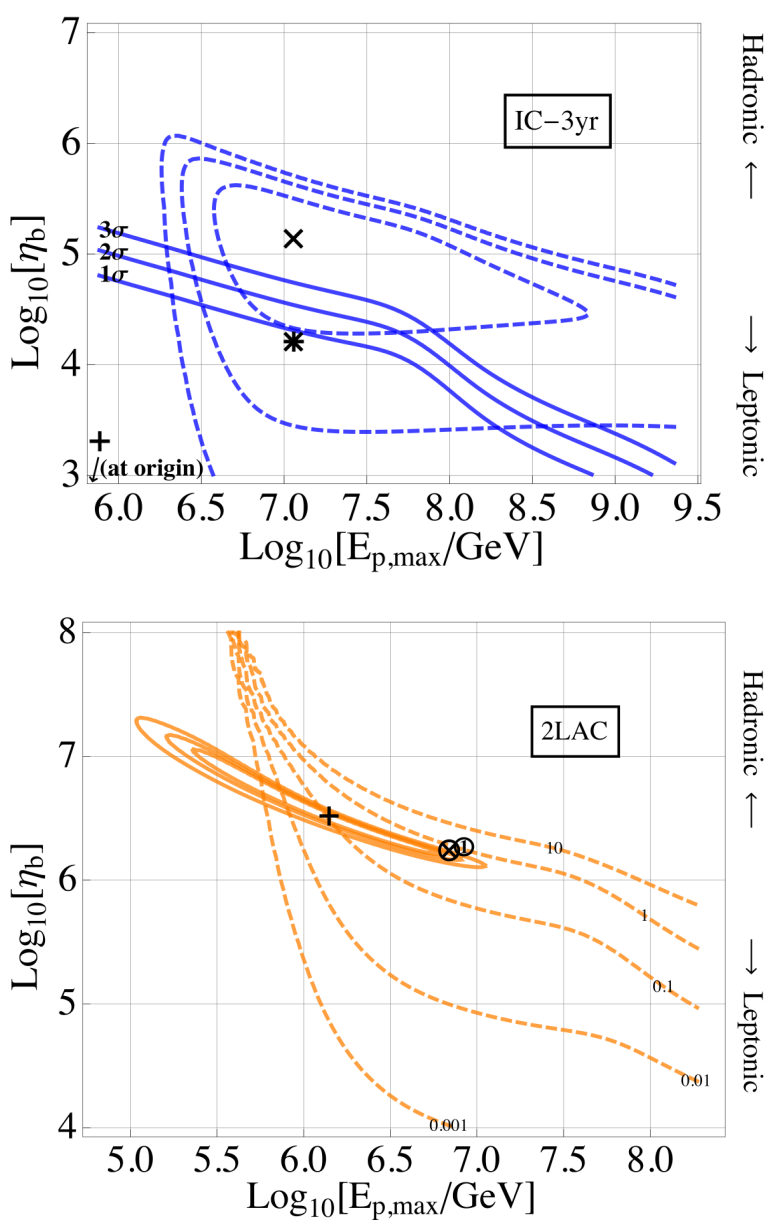

Figure 5. Fit to SED (solid) and neutrino observations (dashed) in terms of maximal proton energy and baryonic loading for the 2LAC (upper panel) and IC-3yr (lower panel) phases (see Fig. 3 for details). In the upper panel, the dashed contours represent the expected number of neutrino events above $0.5 \mathrm{PeV}$, whereas in the lower panel, the same assumptions as for Fig. 3 have been used. Note that the SED best-fit mark + in the lower panel is at the origin since the SED is best reproduced by a pure leptonic model with $\eta_{\mathrm{b}}=0$. See Tab. 2 for the definition of the best-fit symbols.

corrected by this factor of 0.13 , arriving at $\sim 0.2$ events.

\footnotetext{
${ }^{7}$ This is consistent with our prediction of 0.3 events in the $0.5-2.4 \mathrm{PeV}$ bin (Kadler et al. (2016) used the 1.0-2.0 PeV bin) for the joint fit, see Tab. 4.
}

\subsection{Variation in Blazar Activity States}

Let us now address if we can draw a self-consistent picture of the AGN blazar over time. For that purpose, we independently fit the parameters for the three phases 2LAC, IC-3yr, and Burst in Fig. 2; these fits are shown in Fig. 5 for the 2LAC and IC-3yr phases (in addition to Fig. 3 for the Burst phase). Although there is some time overlap, one may interpret these phases in terms of the time evolution of the AGN blazar.

There are two important qualitative differences compared to Fig. 3. First of all, no neutrino candidates have been seen during the 2LAC phase (corresponding to the upper panel) in Fig. 5. We therefore use a different approach for the neutrino expectation: we show the contours of expected number of $>0.5 \mathrm{PeV}$ neutrino events in IceCube during the $2 \mathrm{LAC}$ phase (assuming the same effective area as in the IC-3yr phase) ${ }^{8}$. The best-fit point for the SED in Fig. 5 is marked with "+"; the maximum neutrino flux within the $3 \sigma$ region for the SED fit is marked by " $\otimes$ ", and a representative point on the $N_{\nu}=1$ contour is picked and marked with "(1)". Since the SED of the 2LAC phase has a lower photon flux than that of the IC-3yr or Burst phase, a lower target photon density in the source is implied. In order to have a sufficiently large $p \gamma$ interaction rate to produce the right amount of X-rays and one neutrino event, the required proton-to-lepton luminosity ratio $\eta_{b}$ has to be very large: $\eta_{b} \gtrsim 10^{6}$. From that perspective it is not surprising that no neutrinos were observed during that phase.

The other qualitative difference compared to Fig. 3 concerns the IC-3yr phase (lower panel in Fig. 5). Here we find the SED best-fit favors a pure leptonic SSC model. This means that a baryonic loading is not required for the SED-fit alone. However, within the $3 \sigma$ confidence region of the SED fit, a baryonic loading $\eta_{\mathrm{b}}$ up to $\sim 10^{4.7}$ is allowed without altering the best-fit SED significantly. Taken into account the observed neutrino event occurring during this phase as well, a joint SED and neutrino fit favors a $\eta_{\mathrm{b}} \sim 10^{4.2}$. This example demonstrates that even one neutrino candidate event is able to break the degeneracy of parameters. Together with the multi-

\footnotetext{
${ }^{8}$ Note that IceCube was operational at the time, but only with 40 - and 59string configurations, which would have yielded slightly smaller numbers of neutrinos.
} 


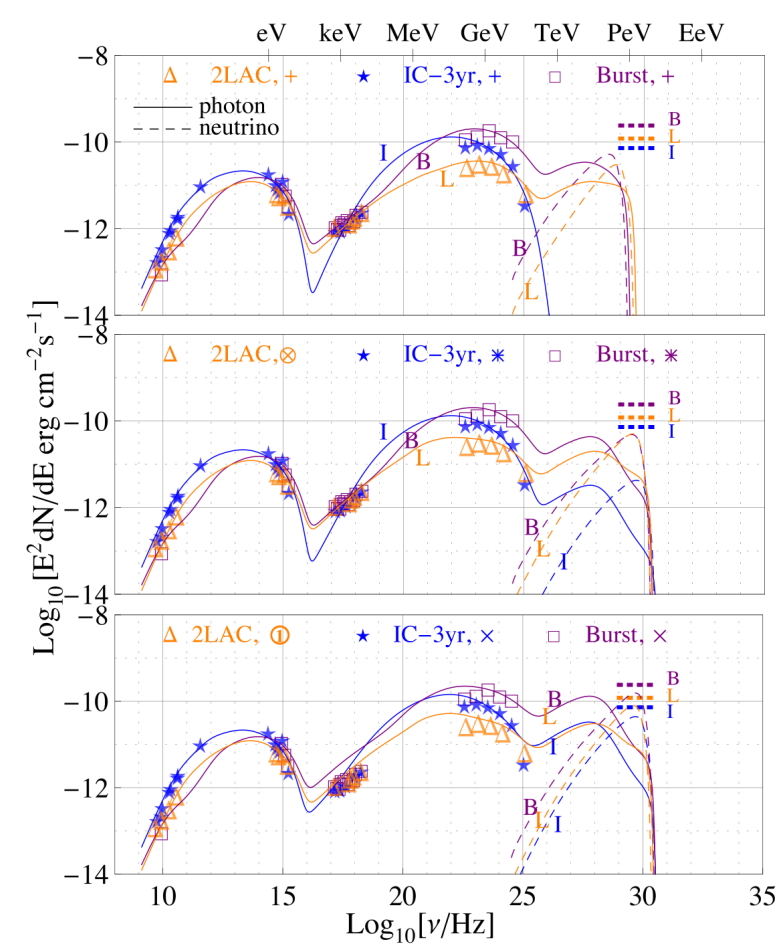

Figure 6. The photon (solid) and all-flavor neutrino (dashed) spectra for the 2LAC (orange,L), IC-3yr (blue,I), and the Burst (purple,B) phases of PKS B1424-418. The upper panel corresponds to the SED best-fit parameters ("+" in Figs. 3 and 5); the middle and lower panels plot the joint (*) and neutrino $(\times)$ best-fit results (except for the 2LAC phase, where we plot the maximum neutrino flux within the $3 \sigma$ contour of SED " $\otimes$ " and one PeV-event flux “(1)"; see Tab. 2.) The horizontal dashed lines around the $\mathrm{PeV}$ energies roughly represent the flux needed to produce one neutrino event in IceCube, given the duration of each phase and IceCube effective area. Note that in the upper panel, the SED best-fit for the IC-3yr phase is a leptonic model and therefore no neutrino is produced. In the bottom panel, due to the lack of IceCube neutrino data during 2LAC period, we have artificially defined the neutrino "best-fit"(1) as the spectrum where one $>0.5 \mathrm{PeV}$-event in IceCube are expected.

waveband data and neutrino observation, our results indicate that $\eta_{\mathrm{b}}$ is lower during this "quiescent" state (IC-3yr) than the active state (Burst).

We finally present the SED for the different phases and different fits (rows) in Fig. 6. We recover that the spectra corresponding to SED (+) and joint (*) fit are also consistent with the SED data by a naked-eye judgement, while in the neutrino fit $(x)$ case an excess in the $X$-ray band is evident. If consistency with the SED is required, however, the neutrino flux is below the flux levels which generate one PeV event in IceCube during the corresponding phases. These are marked by the horizontal-dashed lines in the figure, which are estimated from the duration of each phase and the IceCube effective area at $\mathrm{PeV}$ energies. Most importantly, a self-consistent picture of the SED can be drawn over the different phases if interpreted as variations in blazar properties.

The the X-ray band, seen as the gap between the two humps in the SED, is explained by either leptonic SSC (for IC-3yr phase) or hadronic interactions (for $2 \mathrm{LAC}$, Burst). In the latter case, secondary emission depends on the detailed processing of the electromagnetic cascades in the source, mainly via the following channels: $p \gamma \rightarrow \pi^{0} \rightarrow \gamma \gamma \rightarrow e^{ \pm}$, $p \gamma \rightarrow \pi^{ \pm} \rightarrow \mu^{ \pm} \rightarrow e^{ \pm}$, and $p \gamma \rightarrow p+e^{ \pm}$. In the $\pi^{0}$ channel, the emission predominantly comes from the first generation of pairs. The source itself becomes optically thick for $\gamma$-rays above roughly $100 \mathrm{TeV}$. It can be further estimated Stecker et al. (2012) that the spectrum above $\sim 100 \mathrm{GeV}$ will suffer from additional suppression due to absorption by EBL, but this effect does not play a significantly role in our fitting.

We list the detailed fit parameters and expected number of neutrino events during the different phases in Tab. 4. We notice that similar leptonic parameters are found for the 2LAC, IC-3yr and Burst phases. This suggests that the modeling of the Burst phase can be simply achieved by a slight variation of the magnetic field strength, a slight hardening on the electron injection spectrum, or an increase of the proton injection luminosity. The bulk Lorentz factor, magnetic field values and the blob radius are consistent with the estimates in Eqs. (7)-(8). The spectral index for the power-law injection of primary $e^{-}$is close to -2.0 . The SED and neutrino joint best-fit predicts 0.15 and 0.3 neutrino events in the $>0.5 \mathrm{PeV}$ bins of IceCube during the IC-3yr and Burst phases, respectively. Within the entire $3 \sigma$-confidence regions of the SED fits, up to $\sim 0.4>\mathrm{PeV}$ neutrino events are found during either phase, or about a $5 \%$ chance to have the observed neutrino pattern in IceCube (one 2-PeV event and none in other bins). During the 2LAC phase, within the $3 \sigma$ constraints, we expect at most 0.5 neutrino events above $0.5 \mathrm{PeV}$. The protonto-lepton luminosity ratio required to fit the neutrino result is large, of the order of $10^{4}-10^{5}\left(10^{6}\right.$ for the 2LAC case). This value is coincidently similar to that found for other blazars, such as Diltz et al. (2015); Petropoulou et al. (2015). In our case, on one hand the $\mathrm{p} \gamma$ interaction rate is lower than those in the above cases and more protons are needed to achieve the same level of $\gamma$-rays; on the other hand, compared to their LH $-\pi$ models, we only need a subdominant hadronic contribution to $\gamma$-rays, which lowers the requirement for proton luminosity. The physical proton-injection luminosity needed for the * case roughly equals the Eddington luminosity in the IC-3yr phase and becomes super-Eddington in the Burst phase. This requirement can be alleviated if we assume a lower escape rate for protons, e.g. a magnetic configuration that can trap the protons longer in the blob. In our model, due to the low $p \gamma$ efficiency, most protons simply escape without having a $p \gamma$ interaction. Therefore, the required $L_{\mathrm{p} \text {,inj }}$ scales linearly with the escape rate.

\section{SUMMARY AND CONCLUSIONS}

A potential correlation between the $\mathrm{PeV}$ neutrino event "Big Bird" by IceCube in 2012 and the Burst phase of FSRQ PKS B1424-418 was reported by Kadler et al. (2016). That analysis relies on the frequently used assumption $L_{\gamma} \approx L_{\nu}$. In this paper we have revisited this important relationship 


\begin{tabular}{|c|c|c|c|c|c|c|c|c|c|}
\hline $\begin{array}{l}\text { Phase } \\
\text { Time }\end{array}$ & \multicolumn{3}{|c|}{$\begin{array}{c}\text { 2LAC } \\
2008.9-2010.9\end{array}$} & \multicolumn{3}{|c|}{$\begin{array}{c}\text { IC-3yr } \\
2010.5-2013.5\end{array}$} & \multicolumn{3}{|c|}{$\begin{array}{c}\text { Burst } \\
2012.5-2013.5\end{array}$} \\
\hline $\begin{array}{c}R_{\text {blob }} / \mathrm{cm} \\
\Gamma_{\text {bulk }}\end{array}$ & \multicolumn{9}{|c|}{$\begin{array}{c}7.5 \times 10^{17} \\
35\end{array}$} \\
\hline $\begin{array}{c}B^{\prime} / \mathrm{mG} \\
L_{\mathrm{e}, \mathrm{inj}} / L_{\mathrm{edd}} \\
\gamma_{\mathrm{e}, \min }^{\prime} \\
\gamma_{\mathrm{e}, \max }^{\prime} \\
\alpha_{\mathrm{e}, \mathrm{idx}}^{\prime}\end{array}$ & \multicolumn{3}{|c|}{$\begin{array}{c}3.0 \\
5.9 \times 10^{-6} \\
1.6 \times 10^{3} \\
1.5 \times 10^{5} \\
-2.2\end{array}$} & \multicolumn{3}{|c|}{$\begin{array}{c}3.7 \\
1.7 \times 10^{-5} \\
1.5 \times 10^{3} \\
1.1 \times 10^{5} \\
-2.3\end{array}$} & \multicolumn{3}{|c|}{$\begin{array}{c}2.5 \\
1.7 \times 10^{-5} \\
3.3 \times 10^{3} \\
1.6 \times 10^{5} \\
-2.1\end{array}$} \\
\hline$\alpha_{\mathrm{p}, \mathrm{idx}}^{\prime}$ & \multicolumn{9}{|c|}{-2.0} \\
\hline $\begin{array}{l}\text { Fit symbol } \\
\quad \text { Fit }\end{array}$ & $\begin{array}{c}+ \\
\text { SED }\end{array}$ & $\begin{array}{c}\otimes \\
\text { Tab. } 2\end{array}$ & $\begin{array}{c}\text { (1) } \\
\text { Tab. } 2\end{array}$ & $\begin{array}{c}+ \\
\text { SED }\end{array}$ & $\begin{array}{c}\text { * } \\
\text { joint }\end{array}$ & $\begin{array}{l}\times \\
\nu\end{array}$ & $\begin{array}{c}+ \\
\text { SED }\end{array}$ & $\begin{array}{c}* \\
\text { joint }\end{array}$ & $\begin{array}{l}\times \\
\nu\end{array}$ \\
\hline $\begin{array}{c}\eta_{\mathrm{b}} / 10^{5} \\
E_{\mathrm{p}, \max }^{\mathrm{ob}} / \mathrm{PeV}\end{array}$ & $\begin{array}{c}32 \\
1.67\end{array}$ & $\begin{array}{l}17 \\
8.3\end{array}$ & $\begin{array}{c}18 \\
10.1 \\
\end{array}$ & $\begin{array}{l}0 \\
- \\
\end{array}$ & $\begin{array}{l}0.16 \\
13.7\end{array}$ & $\begin{array}{c}1.3 \\
13.7\end{array}$ & $\begin{array}{c}12 \\
0.92 \\
\end{array}$ & $\begin{array}{l}3.9 \\
8.3\end{array}$ & $\begin{array}{c}5.1 \\
12.4\end{array}$ \\
\hline$N_{\nu}, 0.5-1.6 \mathrm{PeV}$ & 0.11 & 0.51 & 0.74 & 0 & 0.066 & 0.69 & 0.008 & 0.25 & 0.68 \\
\hline$N_{\nu}, 1.6-2.4 \mathrm{PeV}$ & 0 & 0.10 & 0.18 & 0 & 0.017 & 0.18 & 0 & 0.050 & 0.19 \\
\hline$N_{\nu}, \quad>2.4 \mathrm{PeV}$ & 0 & 0.038 & 0.10 & 0 & 0.071 & 0.17 & 0 & 0.017 & 0.15 \\
\hline$P_{0,1,0}\left(E_{\mathrm{p}, \max }, \eta_{\mathrm{b}}\right), \%$ & - & - & - & 0 & 1.6 & 6.4 & 0 & 3.6 & 6.7 \\
\hline photon SED $\chi^{2} /$ d.o.f. & 1.0 & 1.6 & 5.6 & 1.0 & 1.09 & 3.9 & 1.0 & 1.06 & 33 \\
\hline
\end{tabular}

Table 4. Parameters and the expected number of neutrino events in IceCube for the best-fit models during each phase. See Tab. 2 for the definition of symbols and notations.

with a self-consistent one-zone emission model, with SEDs measured from three phases of the source: 2LAC (2008.82010.9), IceCube 3-year (IC-3yr, 2010.5-2013.5) and Burst phase (2012.5-2013.3). Those different SEDs can be interpreted in our results as variations on blazar properties over time. We have also studied the parameter values needed to attribute to the neutrino event, which was observed in the IC$3 \mathrm{yr}$ phase and within that, the Burst phase.

We found that the "conventional" hadronic model (high energy hump by emissions from secondaries via hadronic processes; the "LH- $\pi$ " model) does not work for this blazar, which is different from many other blazars, such as the wellstudied Mrk 421 Dimitrakoudis et al. (2014); Petropoulou et al. (2016), for which the high-energy hump of SED can be fully accounted for by hadronic processes. Therefore, the leading contribution to the SED of PKS B1424-418 must be leptonic. We have, however, shown that a subdominant hadronic contribution can be present. In terms of neutrino production, it is therefore an interesting question how much baryonic loading can be tolerated, even in an SSC model, without affecting the shape of the SED.

We have found that the observed one neutrino event reported by Kadler et al. (2016) during the Burst phase is in tension with the SED fits, whereas up to $\sim 0.3$ above-PeV events are acceptable. The probability to produce 0,1 , and 0 neutrinos in the $0.5-1.6,1.6-2.4$, and $>2.4 \mathrm{PeV}$ bins, respectively, as observed by IceCube, is up to $5 \%$ in both the IC-3yr and the Burst phase. This suggests a chance coincidence of the observed PeV-event with the Burst phase of
PKS B1424-418, since there is a substantial probability for it to occur at any other time of the IceCube 3-year observation. Our predicted event rates are consistent with Kadler et al. (2016) if their expectation is corrected for small fraction of the photon energy flux in the second peak comes from hadronic processes. This means that an additional "theory correction factor" has to be applied if the gamma-ray and neutrino fluxes are to be correlated, which can only come from self-consistent models.

We have also demonstrated from independent simulations of the different phases of the blazar that a self-consistent time-dependent evolution picture can be drawn, meaning that the different phases can be described by similar parameters. We noted that the active state (Burst phase) can host a larger hadronic loading than quiescent phase (IC-3yr). This may as well due to the availability of additional data points during the latter phase, which provides stronger constraints for the fit.

While our results apply to PKS B1424-418 specifically, we emphasize the importance of a self-consistent description of the SED as opposed to to generic approaches relating the gamma-ray and neutrino luminosities. The studied blazar serves as a counter-example for the direct correlation of these, which means that this assumption does not hold in general and has to be used with care. We have also presented an example in a quantitative way on how the neutrino observation, even with one candidate event, is able to break the degeneracies of blazar models. 
SG and MP acknowledge support by the Helmholtz Alliance for Astroparticle Physics HAP funded by the Initiative and Networking Fund of the Helmholtz Association. WW acknowledges funding from the European Research Council (ERC) under the European Union's Horizon 2020 research and innovation programme (Grant No. 646623).

\section{REFERENCES}

Aartsen, M. G., Abbasi, R., Abdou, Y., et al. 2013, Physical Review Letters, 111,021103

Aartsen, M. G., et al. 2015, Astrophys. J., 805, L5

Abbasi, R., et al. 2012, Nature, 484, 351

Ahlers, M., \& Halzen, F. 2014, Phys.Rev.D, 90, 043005

Ajello, M., Gasparrini, D., Sánchez-Conde, M., et al. 2015,

Astrophys.J.Lett., 800, L27

Bechtol, K., Ahlers, M., Di Mauro, M., Ajello, M., \& Vandenbroucke, J. 2015, arXiv: 1511.00688

Blumenthal, G. R. 1970, Phys.Rev.D, 1, 1596

Böttcher, M., Reimer, A., Sweeney, K., \& Prakash, A. 2013, Astrophys.J., 768,54

Bromberg, O., \& Levinson, A. 2009, Astrophys.J., 699, 1274

Chang, J. S., \& Cooper, G. 1970, Journal of Computational Physics, 6, 1

Chen, X., Pohl, M., Böttcher, M., \& Gao, S. 2016, M.N.R.A.S, 458, 3260

Dermer, C. D., Murase, K., \& Inoue, Y. 2014, Journal of High Energy

Astrophysics, 3, 29

Diltz, C., \& Böttcher, M. 2016, Astrophys.J., 826, 54

Diltz, C., Böttcher, M., \& Fossati, G. 2015, Astrophys.J., 802, 133

Dimitrakoudis, S., Petropoulou, M., \& Mastichiadis, A. 2014, Astroparticle Physics, 54, 61

Finke, J. D., Dermer, C. D., \& Böttcher, M. 2008, Astrophys.J., 686, 181

Fossati, G., Maraschi, L., Celotti, A., Comastri, A., \& Ghisellini, G. 1998, M.N.R.A.S, 299,433

Ghisellini, G. 2016, Galaxies, 4, 36

Giannios, D. 2013, M.N.R.A.S, 431, 355
Giannios, D., Uzdensky, D. A., \& Begelman, M. C. 2009, M.N.R.A.S, 395, L29

Glüsenkamp, T. 2016, EPJ Web Conf., 121, 05006

Halzen, F., \& Kheirandish, A. 2016, ArXiv e-prints, arXiv:1605.06119

Hümmer, S., Rüger, M., Spanier, F., \& Winter, W. 2010, Astrophys.J., 721, 630

IceCube Collaboration. 2013, Science, 342, 1242856

Kadler, M., Krauß, F., Mannheim, K., et al. 2016, Nature Physics, 12, 807

Kowalski, M. 2016, talk given at the Neutrino 2016 conference

Krauß, F., Kadler, M., Mannheim, K., et al. 2014, Astron.Astrophys., 566, L7

Lipari, P., Lusignoli, M., \& Meloni, D. 2007, Phys.Rev.D, 75, 123005

Murase, K., Ahlers, M., \& Lacki, B. C. 2013, Phys.Rev.D, 88, 121301

Murase, K., Inoue, Y., \& Dermer, C. D. 2014, Phys.Rev.D, 90, 023007

Padovani, P., Resconi, E., Giommi, P., Arsioli, B., \& Chang, Y. L. 2016, M.N.R.A.S, 457,3582

Petropoulou, M., Coenders, S., \& Dimitrakoudis, S. 2016, Astroparticle Physics, 80, 115

Petropoulou, M., Dimitrakoudis, S., Padovani, P., Mastichiadis, A., \& Resconi, E. 2015, M.N.R.A.S, 448, 2412

Righi, C., Tavecchio, F., \& Guetta, D. 2016, ArXiv e-prints, arXiv: 1607.08061

Stecker, F. W., Malkan, M. A., \& Scully, S. T. 2012, Astrophys.J., 761, 128

Tavecchio, F., Pacciani, L., Donnarumma, I., et al. 2013, M.N.R.A.S, 435, L24

Turley, C. F., et al. 2016, arXiv:1608.08983

\section{APPENDIX}

\section{A. NUMERICAL METHODS}

We simulate time-dependent particle spectra for $e^{ \pm}, p, n, \gamma$, and $\nu_{\alpha}$ ( $\alpha$ denotes the neutrino flavor) by numerically solving integro-differential kinetic equations in energy space,

$$
\frac{\partial n(E)}{\partial t}=\hat{D}(E) n(E)-\alpha(E) n(E)+\epsilon(E),
$$

where $n(E) \equiv d N / d \ln E d V=E d N / d E d V$ is the differential number density of particles and

$$
\hat{D}(E) \equiv-\frac{\partial}{\partial \ln E}\left[A(E)-B(E) \frac{\partial}{\partial \ln E}\right]
$$

is the differential operator that contains terms describing continuous energy gain or loss and momentum diffusion for $e^{ \pm}$and $p$, such as synchrotron emission, inverse Compton scattering in the Thomson regime and Bethe-Heitler losses (in which a proton loses a tiny fraction of energy producing one electron-positron pair). The sink and source terms are written as

$$
\alpha(E)=c \sum_{i} \int \sigma_{i}\left(E, E_{1}\right) n_{i}\left(E_{1}\right) d \ln E_{1}
$$

and

$$
\epsilon(E)=\sum_{i} \int R_{i}\left(E \leftarrow E_{1}\right) n_{i}\left(E_{1}\right) d \ln E_{1}
$$

respectively, and are obtained by a summation over the target particle species $i$ and the integration over the energy $E_{1}$. In the source term, $R\left(E \leftarrow E_{1}\right)$ is the response function, differential in the logarithmic scale. It describes the overall rate of generating a particle of energy $E$ from a parent particle $i$ of energy $E_{1}$. The particle physics aspects are encoded in $R_{i}$ and $\sigma_{i}$, but they can also result from explicit integrations over the current particle distributions, $n_{i}$, adding to the non-linear characteristic of the set of equations. These terms account for the discontinuous processes, such as all the production and absorption processes for $\gamma, \nu$ and 
$n$, the production of $e^{ \pm}$, the redistribution in energy of $e^{ \pm}$on account of IC scattering in the Klein-Nishina regime, along with the redistribution of $p$ and $n$ after $p \gamma$ and $n \gamma$ interactions. In fact, the terms $R_{i}$ and $\sigma_{i}$ in principle contain the full informations for all processes. The finite resolution of our energy discretizations cannot reflect tiny energy shifts though, such as the energy loss of an $e^{-}$after a Thomson scattering, and these must be modeled as continuous energy losses. Our treatment is accurate to the second order, as we compute the two differential coefficients $A(E)$ and $B(E)$ from $R_{i}$. The second-order term $B(E)$ also helps maintaining energy conservation in the system, at least with the "Chang \& Cooper" finite-difference scheme we use Chang \& Cooper (1970).

We assume time-independent injection of $e^{-}$and $p$ with power-law spectra, $d N_{i} / d E=K_{i}\left(E / E_{i, \min }\right)^{\alpha_{i}}$ for $E_{i, \min }<E<E_{i, \max },(i=$ $\left.e^{-}, p\right)$. We do not model the acceleration processes which may operate in a compact acceleration region inside the blob, from which energetic particles are subsequently injected into the blob, explicitly. The blob serves as the radiation-zone, before these particles escape Chen et al. (2016). This scenario is characterized by four free parameters: $E_{i, \min }, E_{i, \max }, K_{i}$, and $\alpha_{i}$ for each $e^{-}$ and $p$. For the protons, the result is insensitive to $E_{\min }$, and we have set $E_{p, \min }=10 \mathrm{GeV}$.

Numerical solutions to Eq. (A1) are obtained by a finite-difference method using the differentiation schemes of Chang \& Cooper Chang \& Cooper (1970) for energy and Crank-Nicolson for time. The Chang \& Cooper scheme maintains accurate energy conservation while the Crank-Nicolson guarantees numerical stability within a rather permissive range of time-steps, $\Delta t$. For ultra-high-energy electrons, which cool rapidly within one time-step, semi-analytical solutions within each $\Delta t$ were used for efficiency reasons. For $p \gamma$ interactions we compute the pion spectrum and the corresponding coefficients in Eq. (A1) by the method of Sim A in Hümmer et al. (2010). The spectrum of the daughter particles, namely $\mu^{ \pm}, e^{ \pm}$and $\nu$ s, are calculated via the Eqs. (14) to (21) of Lipari et al. (2007). For Bethe-Heitler interaction we constructed a similar framework as Hümmer et al. (2010) from the differential cross-sections Blumenthal (1970) and subsequently computed the coefficients suitable for our Eq. (A1), which again guarantees computational efficiency. The cooling of intermediate particles $\pi^{ \pm}$and $\mu^{ \pm}$prior to their decay can be safely neglected here due to the weak magnetic field. For example, for $B \sim 3 \mathrm{mG}$ and $\mathrm{Y}_{\mathrm{SSC}} \sim 3$, radiative cooling dominates over decay loss at $\gamma_{\mu}>10^{13}$ for $\mu^{ \pm}$and $\gamma_{\pi}^{\prime}>10^{14}$ for $\pi^{ \pm}$, which is well beyond the value of $E_{p \text {, max }}^{\prime}$. For $e^{-}$, cooling dominates over escape at $\gamma_{\mathrm{e}}^{\prime}>10^{6}$. 